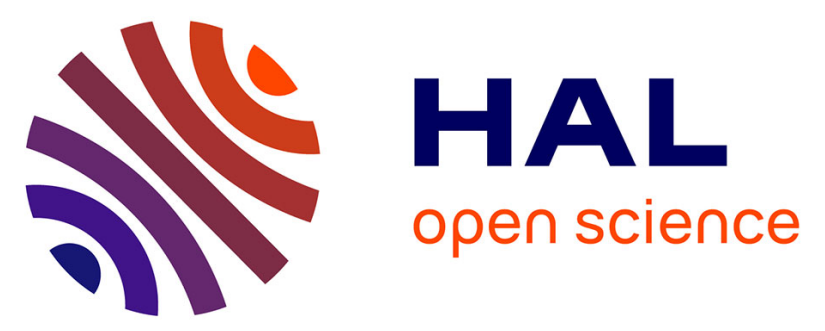

\title{
Influence des hautes températures des racines sur la croissance de plants de diverses variétés de melon (Cucumis melo L.). Aspects particuliers concernant la nutrition de la plante
}

Georgette Risser, Pierre Cornillon, Jean-Claude Rode, Magali Auge, Paul

Florent, Andrée Ruffinato

\section{To cite this version:}

Georgette Risser, Pierre Cornillon, Jean-Claude Rode, Magali Auge, Paul Florent, et al.. Influence des hautes températures des racines sur la croissance de plants de diverses variétés de melon (Cucumis melo L.). Aspects particuliers concernant la nutrition de la plante. Agronomie, 1983, 3 (8), pp.735-744. hal-00884566

\section{HAL Id: hal-00884566 https://hal.science/hal-00884566}

Submitted on 1 Jan 1983

HAL is a multi-disciplinary open access archive for the deposit and dissemination of scientific research documents, whether they are published or not. The documents may come from teaching and research institutions in France or abroad, or from public or private research centers.
L'archive ouverte pluridisciplinaire HAL, est destinée au dépôt et à la diffusion de documents scientifiques de niveau recherche, publiés ou non, émanant des établissements d'enseignement et de recherche français ou étrangers, des laboratoires publics ou privés. 


\title{
Influence des hautes températures des racines sur la croissance de plants de diverses variétés de melon (Cucumis melo L.). Aspects particuliers concernant la nutrition de la plante
}

\author{
Georgette RISSER \& Pierre CORNILLON * \\ avec la collaboration technique de Jean-Claude RODE, Magali AUGE*, Paul FLORENT* \& Andrée \\ RUFFINATO \\ I.N.R.A., Station d'Amélioration des Plantes maraîchères, Domaine Saint-Maurice. \\ *I.N.R.A., Station d'Agronomie, Domaine Saint-Paul, Centre de Recherches Agronomiques d'Avignon \\ B.P. 94, F 84140 Montfavet
}

De jeunes plants de 4 variétés de melon ont été cultivés en solution nutritive maintenue à diverses températures entre $18^{\circ} \mathrm{C}$ et $34^{\circ} \mathrm{C}$ alors que la température de l'air ambiant était inférieure à $30^{\circ} \mathrm{C}$, à 2 périodes : fin d'hiver et début d'été.

Aucun effet systématique de la température racinaire n'a été observé en période estivale alors qu'en période de faible rayonnement, la croissance était d'autant meilleure que la température racinaire était plus élevée, sauf pour la variété «Persian Small Type ».

De fortes différences variétales existent en effet en période de faible rayonnement global. « Freeman's cucumber» profite mieux de l'augmentation de la température racinaire que les types «Charentais »: «Doublon» et "Védrantais", alors qu'au-dessus de $22^{\circ} \mathrm{C}$ l'augmentation de la température racinaire est nuisible à la croissance de "Persian Small Type». Dans les conditions de l'essai, le maintien d'une température racinaire supérieure à celle de l'air n'a pas entraîné d'anomalie de croissance.

Au-dessus d'une température racinaire de $18{ }^{\circ} \mathrm{C}$, le rayonnement global devient le facteur limitant essentiel de la croissance du melon.

Mots clés additionnels: Cucurbitacées, température racinaire, nutrition minérale, nutrition hydrique, rayonnement global, variabilité génétique. Particular aspects concerning the nutrition's plant.

Young plants of 4 muskmelon varieties were cultivated in nutrient solution with constant root temperature between $18^{\circ} \mathrm{C}$ and $34^{\circ} \mathrm{C}$ and air temperature below $30^{\circ} \mathrm{C}$ at two times of year : end of winter and beginning of summer.

Provided root temperature was always above air temperature, muskmelon growth was normal. Root temperature had no effect in summer but at the end of winter higher root temperature gave better growth. There were great varietal differences during this period of low total radiation. "Freeman's cucumber" grew better than the "Charentais" varieties: "Doublon" and "Védrantais", when the root temperature was high ; for "Persian Small Type", root temperatures higher than $22{ }^{\circ} \mathrm{C}$ were unfavourable.

At root temperatures higher than $18^{\circ} \mathrm{C}$, total radiation was the main factor limiting muskmelon growth.

Additional key words: Cucumis melo L. Cucurbitaceae, root temperature, high temperature, mineral nutrition, water supply, total radiation, genetic variability.

\section{INTRODUCTION}

L'utilisation de paillage plastique transparent s'est généralisée dans les cultures de melon car cette technique permet d'obtenir un gain de précocité intéressant et de retarder les contaminations virales.
Actuellement, plus de 90 p. 100 des cultures de melon sont conduites avec cette technique qui occasionne une élévation très importante de la température du sol. Cette modification du micro-climat transforme les conditions de croissance et de nutrition de la plante. Sous serre, le développement des cultures hors sol modifie également le 
niveau des températures racinaires pendant toute la durée de la culture.

Aussi, pour apporter des éléments de réponse aux problèmes soulevés par les températures élevées observées au niveau des racines, nous avons entrepris une étude de ce facteur en culture hydroponique. L'utilisation de cette technique permet d'analyser les effets directs d'une température des racines supérieure à celle de la partie aérienne et de suivre la cinétique de nutrition des plantes.

Après avoir précisé les conditions expérimentales des 2 essais successifs conduits dans des conditions de photopériodes différentes, nous présentons successivement les résultats concernant la croissance de la plante et ceux ayant trait à la nutrition hydrique et minérale.

\section{MATÉRIEL ET MÉTHODES}

\section{A. Variétés et températures}

Les essais ont été faits sur 3 variétés déjà présentées dans des essais antérieurs (RISSER et al., 1978): "Doublon» (origine I.N.R.A.-France), «Védrantais» (origine Vilmorin-France), «Persian Small Type » (origine Université du Michigan-U.S.A.) ; la $4^{e}$ variété, "Freeman's cucumber» (origine asiatique, via Israël) a été choisie pour son excellent comportement à basse température dans un essai de culture sous abri non chauffé (RISSER, 1979).

Les 4 températures du substrat expérimentées ont été $18^{\circ}$, $22^{\circ}, 26^{\circ}$ et $30^{\circ} \mathrm{C}$ dans le $1^{\text {er }}$ essai ; $22^{\circ}, 26^{\circ}, 30^{\circ}$ et $34^{\circ} \mathrm{C}$ dans le $2^{\mathrm{e}}$ avec une précision de $\pm 0,5^{\circ} \mathrm{C}$. Cet essai nous a permis d'observer l'influence d'une température racinaire supérieure à celle de l'air sur la croissance du melon.

\section{B. Conduite des essais}

Les autres caractéristiques climatiques sont récapitulées dans le tableau 1 qui montre notamment que le facteur climatique ayant le plus varié entre les 2 essais, est le rayonnement global.
Les essais ont été réalisés en serre chauffée vitrée, avec un système expérimental et une solution nutritive déjà décrite (CORNILlon \& Boudon, 1979). A la fin du $2^{\mathrm{e}}$ essai, une modification accidentelle s'est produite, ce qui a conduit à une solution nutritive dont la concentration en $\mathrm{PO}_{4} \mathrm{H}_{2} \mathrm{NH}_{4}$ était inférieure de moitié à celle de la concentration théorique. Les plantes de melon préalablement élevées dans du sable stérilisé ont été placées dans le dispositif au stade cotylédons étalés, la température étant maintenue uniformément à $22{ }^{\circ} \mathrm{C}$ pendant quelques jours pour permettre une bonne reprise des plantules avant le réglage définitif des températures.

Huit répétitions de 1 plante par variété étaient observées par température, chaque plante disposant de 1 litre de solution nutritive.

\section{Mesures}

\section{Surface des feuilles}

La surface des feuilles a été mesurée avec un planimètre optique d'une précision au moins égale à $0,5 \mathrm{~cm}^{2}$.

\section{Analyse minérale}

Nous avons successivement analysé les solutions nutritives et les plantes. Des échantillons sont prélevés à chaque renouvellement de la solution nutritive.

Les concentrations en ions nitrate et ammonium ainsi que le $\mathrm{pH}$ sont mesurés avec une électrode spécifique. Le phosphore est déterminé par colorimétrie au vanadomolybdate, le potassium par photométrie de flamme et le calcium et le magnésium par spectrophotométrie d'absorption atomique.

La détermination des éléments minéraux dans les tissus végétaux est opérée en utilisant les méthodes mises au point par le Comité Inter Institut (C.I.I., 1973). Ces déterminations sont effectuées sur les organes séparés et lavés au moment de la récolte puis fixés pour éviter toute évolution ultérieure.

\section{TABLEAU 1}

Caractérisation climatique de la période des essais. Climatic characteristics during the experimental period.

\begin{tabular}{|c|c|c|c|c|c|c|}
\hline \multirow[b]{3}{*}{ Essai } & \multicolumn{4}{|c|}{ Valeur journalière } & \multicolumn{2}{|c|}{ Total } \\
\hline & \multicolumn{2}{|c|}{ minimum } & \multicolumn{2}{|c|}{ maximum } & \multirow[b]{2}{*}{ (1) } & \multirow[b]{2}{*}{ (2) } \\
\hline & (1) & (2) & (1) & (2) & & \\
\hline $\begin{aligned} \text { Température de l'air sous serre } & \\
& =\text { maximum } \\
& =\text { minimum } \\
& =\varepsilon \text { des températures } \\
& \left(>12^{\circ} \mathrm{C}\right) \\
& \left(>0^{\circ} \mathrm{C}\right)\end{aligned}$ & $\begin{array}{r}26 \\
14\end{array}$ & $\begin{array}{l}18 \\
16\end{array}$ & $\begin{array}{l}32 \\
16\end{array}$ & $\begin{array}{l}33 \\
20\end{array}$ & $\begin{array}{l}179 \\
407\end{array}$ & $\begin{array}{l}173 \\
353\end{array}$ \\
\hline Duréc insolation en $h$ & 0,0 & 4,7 & 10,7 & 14,0 & 127 & 165 \\
\hline Rayonnement global MJ.m-2 extérieur & 3,6 & 11,2 & 16,3 & 27,6 & 208 & 342 \\
\hline
\end{tabular}

(1) $1^{\text {er }}$ essai : 19 février-9 mars, soit $18 \mathrm{j}$.

(2) $2^{\mathrm{e}}$ essai : 19 juin-3 juillet, soit $14 \mathrm{j}$. 


\section{RÉSULTATS}

\section{A. Croissance de la plante}

Dans le $1^{\text {er }}$ essai qui s'est déroulé pendant une période de faible rayonnement global, certaines plantes ont présenté des anomalies: décoloration des vieilles feuilles de la variété «Védrantais», aspect terne et souffreteux des plantes de «Persian», décolorations en mosaïque à $26^{\circ}$ et $30^{\circ} \mathrm{C}$ de la variété « Freeman's"; la variété « Doublon» a eu un comportement normal. Aucune anomalie n'a été observée au cours du $2^{\mathrm{c}}$ essai.

Nous avons caractérisé la croissance de la plante par un certain nombre de paramètres: nombre de feuilles sur la tige principale, nombre de ramifications secondaires, surface foliaire, poids sec de la plante entière, importance relative des parties aériennes et des racines et teneur en matière sèche du feuillage (tabl. 2).

De l'observation du tableau 2 qui présente les valeurs obtenues en fin d'essai, il ressort que, dans l'essai 1, en période de faible rayonnement, l'élévation de la température racinaire a eu un effet favorable sur le nombre de feuilles et de ramifications, la surface foliaire, le poids sec, le rapport partie aérienne/partie racinaire chez les 3 variétés «Doublon », « Freeman's » et « Védrantais »; chez « Persian ", les valeurs maximales de surface foliaire et de poids sec sont observées à $22^{\circ} \mathrm{C}$. La teneur en matière sèche est plus forte à $18^{\circ} \mathrm{C}$ qu'aux températures plus élevées, la variété «Persian» faisant là encore exception. Dans l'essai 2, aucun effet systématique de la température n'est mis en évidence.

Les différences entre les 2 essais sont importantes : ainsi, en période de fort rayonnement (essai 2), le poids sec est plus élevé, le rapport partie aérienne/partie racinaire plus faible mais la comparaison des 2 essais doit tenir compte de leur durée différente $(18 \mathrm{j}$. pour l'essai $1,14 \mathrm{j}$. pour l'essai 2): ainsi, si en fin d'essai, le tableau 2 montre un nombre de feuilles plus élevé dans l'essai 1, on observe en fait, pour ce caractère qui a été noté régulièrement en cours d'essai, une vitesse d'apparition des feuilles un peu plus rapide dans le $2^{\mathrm{e}}$ essai que dans le $1^{\text {er }}$; il en est de même pour les ramifications.

On note donc dans nos essais une influence prépondérante du rayonnement global sur la croissance des plants de melon ; par contre, les effets de la température des racines des variétés sont beaucoup plus réduits.

\section{B. Consommation hydrique}

A chaque renouvellement de solution nutritive, on apportait $1 \mathrm{l}$. de solution par pot après aspiration du restant de la solution antérieure. La quantité totale de liquide recueillie par variété et par température était mesurée, ce qui permettait de calculer par déduction la quantité d'eau absorbée par les plantes depuis le précédent changement de solution.

La figure 1 présente les consommations hydriques totales (durée complète de l'essai), le tableau 3 les consommations hydriques journalières en fin d'essai, par $\mathrm{g}$ de poids sec et $\mathrm{mm}^{2}$ de surface foliaire.

Les consommations hydriques totales ont été très supérieures dans l'essai 2 alors que, calculées en fonction du poids $\mathrm{sec}$, elles sont légèrement supérieures dans l'essai 1.

La température racinaire n'a pas d'effet sur la consommation hydrique journalière rapportée au poids sec ou à la surface foliaire : l'augmentation de la consommation hydri-

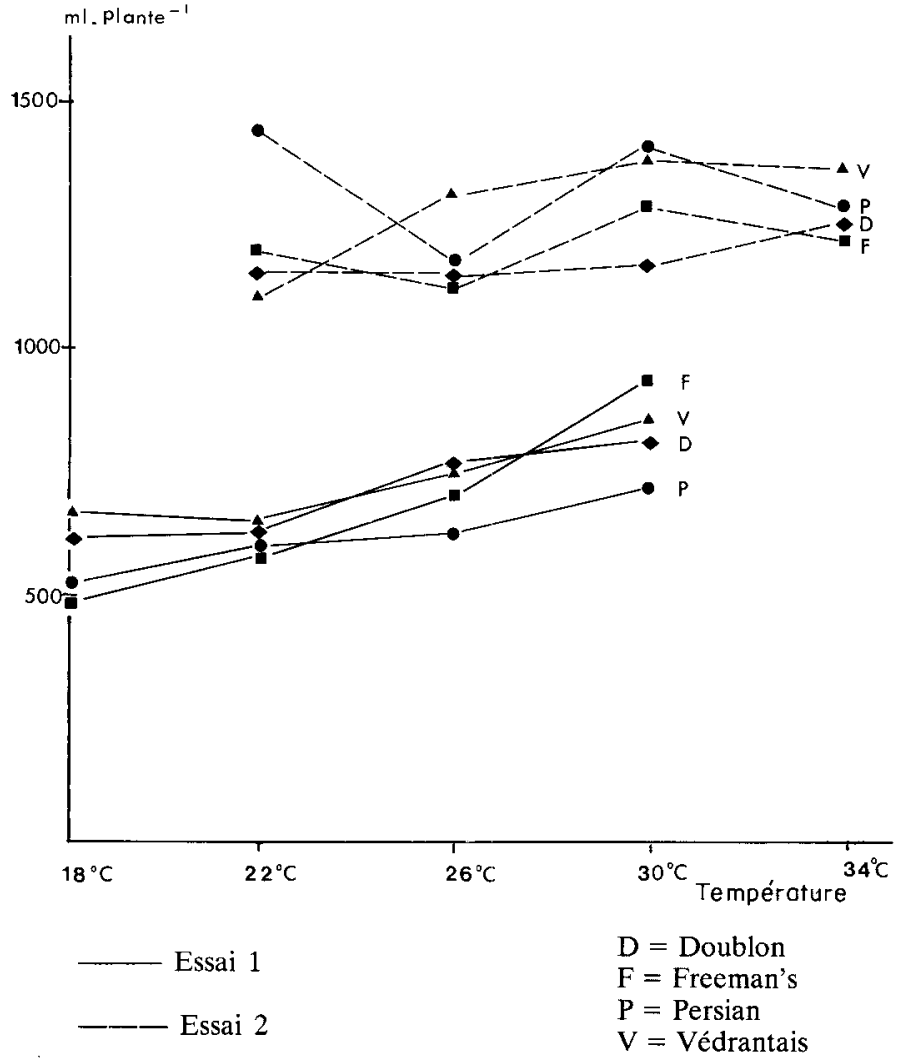

Figure 1

Consommation hydrique totale (moyenne par plante en $\mathrm{ml}$ ). Total water uptake (mllplant).

que totale, observée dans l'essai 1 avec l'élévation de la température racinaire, est liée à l'effet favorable de cette dernière sur la croissance générale de la plante.

En période de faible rayonnement, on observe des consommations d'eau moindres pour « Persian Small Type " et «Freeman's cucumber », si celles-ci sont rapportées à la surface foliaire, et seulement pour «Freeman's cucumber », si elles sont rapportées au poids sec.

\section{Nutrition minérale}

A chaque renouvellement de la solution nutritive, un échantillon de la solution restante était analysé, permettant par comparaison avec la composition de la solution de base de mettre en évidence l'absorption de chacun des éléments.

A la fin de l'essai 2, la composition minérale de la matière sèche a été déterminée.

\section{Evolution de la solution nutritive}

Le tableau 4 présente les variations de composition de la solution, toutes températures et variétés confondues.

Les variations positives indiquent que les plantes ont absorbé plus facilement l'eau que l'élément minéral ; les variations négatives indiquent l'inverse.

Le rapport quantité de nitrate absorbé/volume d'eau consommée, pour chaque variété et température, est présenté dans la figure 2.

\section{a) Phosphore}

L'absorption différenticlle de $\mathrm{PO}_{4} \mathrm{H}_{2}{ }^{-}$est très favorisćc par le fort rayonnement dès le début des essais (tabl. 4). Il est légèrement favorisé par l'augmentation de la température racinaire dans l'essai 1, essai dans lequel « Frecman's cucumber» se distingue des autres variétés par une plus 


\section{TABLEAU 2}

Valeur moyenne a'e quelques caractéristiques de la croissance en fin d'essai (lorsque les différences variétales sont significatives au seuil $5 \%$, elles sont surmontées d'un astérisque).

Value of growth characteristics at the end of the experiment (asterisks indicate significant differences between varietal means).

\begin{tabular}{|c|c|c|c|c|c|c|c|}
\hline Caractéristique & Essai & Variété & $18^{\circ} \mathrm{C}$ & $22{ }^{\circ} \mathrm{C}$ & $\begin{array}{l}\text { Températures } \\
26^{\circ} \mathrm{C}\end{array}$ & $30^{\circ} \mathrm{C}$ & $34^{\circ} \mathrm{C}$ \\
\hline & & & & & & * & \\
\hline \multirow[t]{9}{*}{ Nombre de feuilles sur la tige principale } & 1 & Doublon & 6,6 & 6,5 & 7,0 & 8,0 & \\
\hline & & Freeman's & 6,1 & 6,9 & 7,5 & 8,6 & \\
\hline & & Persian & 6,4 & 7,0 & 7,0 & 7,3 & \\
\hline & & Védrantais & 6,1 & 6,5 & 6,4 & $\begin{array}{c}6,8 \\
*\end{array}$ & \\
\hline & 2 & Doublon & & 5,4 & 6,3 & 5,5 & 5,9 \\
\hline & & Freeman's & & 5,6 & 5,8 & 5,6 & 5,9 \\
\hline & & Persian & & 6,3 & 6,4 & 6,9 & 6,8 \\
\hline & & Védrantais & & 6,0 & 6,0 & 5,9 & 5,9 \\
\hline & & & & & $*$ & * & \\
\hline \multirow[t]{9}{*}{ Nombre de ramifications secondaires } & 1 & Doublon & 0,6 & 0,6 & 1,5 & 2,1 & \\
\hline & & Freeman's & 0,0 & 0,3 & 0,0 & 1,1 & \\
\hline & & Persian & 0,0 & 0,0 & 0,0 & 0,0 & \\
\hline & & Védrantais & 0,6 & 0,6 & $\underset{*}{1,0}$ & 1,3 & $*$ \\
\hline & 2 & Doublon & & 2,0 & 1,9 & 1,5 & 2,5 \\
\hline & & Freeman's & & 0,6 & 0,6 & 1,3 & 0,4 \\
\hline & & Persian & & 1,6 & 0,6 & 1,4 & 1,5 \\
\hline & & Védrantais & & 1,8 & 1,6 & 1,8 & 1,9 \\
\hline & & & & $*$ & $*$ & & \\
\hline \multirow[t]{5}{*}{ Surface foliaire $\left(\mathrm{cm}^{2}\right)$} & 1 & Doublon & 197 & 258 & 331 & 387 & \\
\hline & & Freeman's & 238 & 376 & 472 & 592 & \\
\hline & & Persian & 246 & 350 & 337 & 288 & \\
\hline & & Védrantais & 231 & 248 & 303 & 374 & \\
\hline & & & & $*$ & $*$ & $*$ & \\
\hline \multirow[t]{9}{*}{ Poids sec (g par plante) } & 1 & Doublon & 0,95 & 1,18 & 1,43 & 1,68 & \\
\hline & & Freeman's & 1,06 & 1,58 & 1,86 & 2,38 & \\
\hline & & Persian & 0,75 & 1,19 & 1,09 & 0,99 & \\
\hline & & Védrantais & 1,11 & 1,20 & $\begin{array}{c}1,33 \\
*\end{array}$ & 1,61 & * \\
\hline & 2 & Doublon & & 2,69 & 2,68 & 2,34 & 2,85 \\
\hline & & Freeman's & & 3,07 & 2,24 & 2,94 & 2,29 \\
\hline & & Persian & & 3,85 & 2,94 & 3,74 & 3,18 \\
\hline & & Védrantais & & 2,94 & 3,22 & 2,81 & 3,15 \\
\hline & & & $*$ & * & $*$ & $*$ & \\
\hline \multirow[t]{9}{*}{ Rapport partie aérienne/racine } & 1 & Doublon & 5,33 & 4,90 & 5,50 & 7,00 & \\
\hline & & Freeman's & 5,62 & 7,78 & 9,54 & 10,33 & \\
\hline & & Persian & 9,71 & 10,90 & 9,90 & 13,14 & \\
\hline & & Védrantais & 5,53 & $\begin{array}{c}6,06 \\
*\end{array}$ & $\begin{array}{c}5,65 \\
*\end{array}$ & $\begin{array}{c}7,47 \\
*\end{array}$ & $*$ \\
\hline & 2 & Doublon & & 3,20 & 3,25 & 3,88 & 4,18 \\
\hline & & Freeman's & & 4,68 & 5,79 & 6,17 & 6,63 \\
\hline & & Persian & & 4,58 & 5,25 & 6,06 & 6,23 \\
\hline & & Védrantais & & 3,08 & 3,47 & 4,30 & 4,73 \\
\hline & & & * & * & * & & \\
\hline \multirow{9}{*}{$\begin{array}{l}\text { Teneur en matière sèche (\% de matière } \\
\text { fraîche) }\end{array}$} & & & & & & & \\
\hline & 1 & Doublon & 7,6 & 6,8 & 6,7 & 6,8 & \\
\hline & & Freeman's & 8,5 & 7,8 & 7,3 & 7,2 & \\
\hline & & Persian & 6,4 & 6,6 & 6,2 & 6,9 & \\
\hline & & Védrantais & 7,3 & 6,8 & 6,5 & $\underset{*}{7,1}$ & \\
\hline & 2 & Doublon & & 7,1 & 6,3 & 6,9 & 6,7 \\
\hline & & Freeman's & & 7,7 & 6,8 & 6,8 & 6.5 \\
\hline & & Persian & & 6,9 & 6,6 & 7,3 & 6,9 \\
\hline & & Védrantais & & 7,0 & 6,4 & 6,9 & 6,6 \\
\hline
\end{tabular}


TABLEAU 3

Consommation hydrique journalière en fin d'essai. (E1: période du 7/3 au 9/3; E2: période du $30 / 6$ au 3/7).

Daily water uptake at the end of the experiment. (E1: period from $7 / 3$ to $9 / 3 ; E 2:$ period from $30 / 6$ to $3 / 7$ ).

\begin{tabular}{|c|c|c|c|c|c|c|c|c|c|c|c|c|}
\hline & & \multicolumn{6}{|c|}{ Par gramme de poids sec } & \multicolumn{5}{|c|}{ Par $\mathrm{mm}^{2}$ de surface foliaire } \\
\hline & & $18^{\circ} \mathrm{C}$ & $22^{\circ} \mathrm{C}$ & $26^{\circ} \mathrm{C}$ & $30^{\circ} \mathrm{C}$ & $34^{\circ} \mathrm{C}$ & $\begin{array}{c}\left({ }^{*}\right) \\
\text { Moyen. }\end{array}$ & $18^{\circ} \mathrm{C}$ & $22^{\circ} \mathrm{C}$ & $26^{\circ} \mathrm{C}$ & $30^{\circ} \mathrm{C}$ & $\begin{array}{c}(*) \\
\text { Moyen }\end{array}$ \\
\hline Doublon & $\begin{array}{l}\mathrm{E} 1 \\
\mathrm{E} 2\end{array}$ & 80 & $\begin{array}{l}69 \\
58\end{array}$ & $\begin{array}{l}74 \\
59\end{array}$ & $\begin{array}{l}68 \\
65\end{array}$ & 63 & $\begin{array}{l}73 \\
61\end{array}$ & 38 & 31 & 32 & 29 & 33 \\
\hline Védrantais & $\begin{array}{l}\text { E1 } \\
\text { E2 }\end{array}$ & 67 & $\begin{array}{l}70 \\
56\end{array}$ & $\begin{array}{l}73 \\
60\end{array}$ & $\begin{array}{l}71 \\
66\end{array}$ & 62 & $\begin{array}{l}70 \\
61\end{array}$ & 32 & 34 & 32 & 31 & 32 \\
\hline Persian & $\begin{array}{l}\text { E1 } \\
\text { E2 }\end{array}$ & 79 & $\begin{array}{l}64 \\
59\end{array}$ & $\begin{array}{l}65 \\
58\end{array}$ & $\begin{array}{l}77 \\
58\end{array}$ & 56 & $\begin{array}{l}71 \\
58\end{array}$ & 24 & 22 & 21 & 27 & 24 \\
\hline Freeman's & $\begin{array}{l}\mathrm{E} 1 \\
\mathrm{E} 2\end{array}$ & 58 & $\begin{array}{l}56 \\
54\end{array}$ & $\begin{array}{l}57 \\
58\end{array}$ & $\begin{array}{l}58 \\
60\end{array}$ & 59 & $\begin{array}{l}57 \\
58\end{array}$ & 25 & 24 & 23 & 23 & 24 \\
\hline $\begin{array}{l}\text { Moyenne } \\
\text { température }\end{array}$ & $\begin{array}{l}\text { E1 } \\
\text { E2 }\end{array}$ & 71 & $\begin{array}{l}65 \\
57\end{array}$ & $\begin{array}{l}67 \\
59\end{array}$ & $\begin{array}{l}69 \\
62\end{array}$ & 60 & & 30 & 28 & 27 & 28 & \\
\hline
\end{tabular}

$\left({ }^{*}\right)$ : Moyenne variétale.

\section{TABLEAU 4}

Evolution de la solution nutritive, toutes températures et variétés confondues (valeur moyenne finale moins valeur initiale de la teneur en divers éléments en $\left.\mathrm{mg} . \mathrm{l}^{-1}\right)$.

Change in nutrient solution composition, for all temperatures and varieties confounded (mean final value minus initial value for each nutrient).

\begin{tabular}{|c|c|c|c|c|c|c|c|c|c|}
\hline Essai : & & & Essa & & & & & Essai 2 & \\
\hline $\begin{array}{l}\text { Date de renouvellement de la solution (en } \mathrm{j} \\
\text { après début essai) }\end{array}$ & 9 & 11 & 14 & 16 & 18 & 4 & 8 & 11 & 14 \\
\hline $\mathrm{PO}_{4} \mathrm{H}_{2}^{-}$ & $-1,9$ & $-3,5$ & $-3,0$ & $-7,2$ & $-7,4$ & $-20,4$ & $-25,0$ & $-10,9^{\circ}$ & $-10,9^{\circ}$ \\
\hline $\mathrm{NO}_{3}^{-}$ & $+0,1$ & 0 & $-0,3$ & $-0,2$ & $-0,4$ & $+0,1$ & $+0,3$ & $-1,2$ & $-\quad 5,0$ \\
\hline $\mathrm{K}^{+}$ & +17 & -11 & -17 & -14 & -3 & +7 & -11 & -36 & -104 \\
\hline $\mathrm{Mg}^{++}$ & $-0,6$ & $-0,2$ & $+1,4$ & $+0,7$ & $-0,6$ & $-0,2$ & $-0,3$ & $-4,0$ & $-\quad 5,3$ \\
\hline $\mathrm{Ca}^{++}$ & $-0,8$ & $-0,3$ & $+4,5$ & $-7,8$ & $-1,8$ & +1 & -7 & -9 & +52 \\
\hline
\end{tabular}

- Solution épuisée, concentration initiale anormalement basse.

forte absorption préférentielle de $\mathrm{PO}_{4} \mathrm{H}_{2}^{-}$. Dans l'essai 2, aucun effet du facteur température ou variété n'a pu être mis en évidence.

$$
\text { b) } \mathrm{NO}_{3}^{-}
$$

$\mathrm{NO}_{3}^{-}$est très légèrement moins absorbé que l'eau en début de croissance puis l'absorption devient de plus en plus forte, surtout dans l'essai 2 (tabl. 4). La température racinaire n'a pas eu d'effet notable ; par contre on observe un net effet variétal, "Freeman's cucumber » absorbant plus et
"Persian Small Type » moins de $\mathrm{NO}_{3}^{-}$que les variétés "Doublon " et "Védrantais " dans l'essai 1. Dans l'essai 2, par contre, c'est «Persian Small Type » qui absorbe le mieux l'ion $\mathrm{NO}_{3}{ }^{-}$.

L'expression de l'absorption des nitrates par litre d'eau consommé est maximum à $22^{\circ} \mathrm{C}$ puis décroît quand la température augmente pour l'ensemble des observations. D'autre part, l'effet du rayonnement n'apparaît pas, sauf pour la variété «Freeman's » pour laquelle les valeurs les plus élevées correspondent au rayonnement le plus faible (Essai 1). 


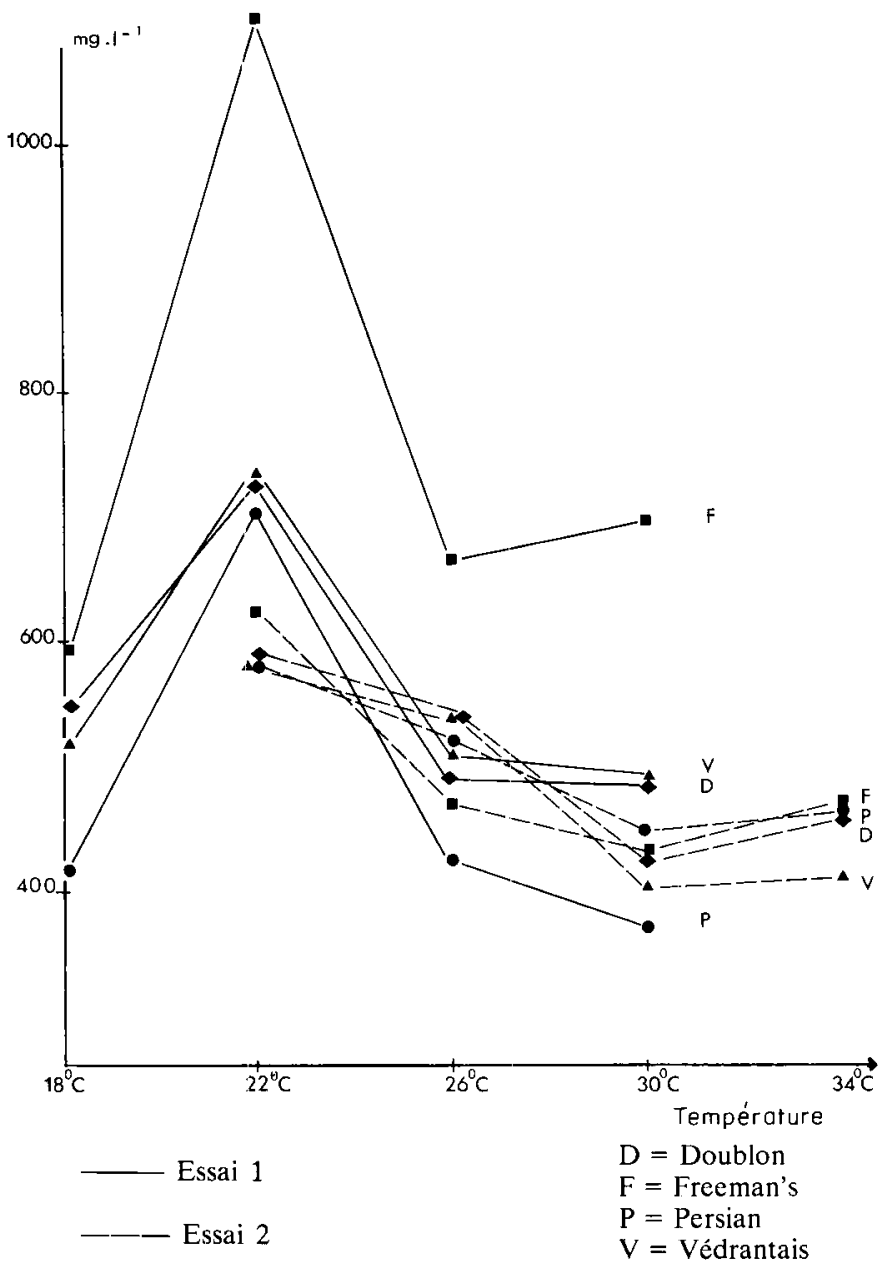

Figure 2

Influence de la température des racines sur l'absorption des nitrates par 4 variétés de melon (mg par litre d'eau consommé).

Nitrate uptake $\left(m g / l^{-1}\right)$.

Nous ne notons pas de corrélation apparente entre la croissance et la concentration en nitrates à l'absorption. Ces résultats suggèrent que la croissance du melon ne dépend pas directement de l'action des conditions de température de racines et du rayonnement sur la nutrition azotée.

$$
\text { c) } \mathrm{NH}_{4}^{+}
$$

La quantité d'ions $\mathrm{NH}_{4}^{+}$n'a été mesurée que dans l'essai 2. L'absorption de $\mathrm{NH}_{4}{ }^{+}$diminue légèrement avec l'augmentation de la température racinaire. "Persian Small Type " semble un pcu mieux absorber $\mathrm{NH}_{4}{ }^{+}$que les autres variétés.

\section{d) Potassium}

Dans les 2 essais, on constate la même évolution au cours du temps: enrichissement de la solution en potassium en début de croissance puis appauvrissement de plus en plus important surtout dans l'essai 2; en dćbut d'essais, le potassium est un peu mieux absorbé aux basses températures. De nettes différences variétales existent dans l'essai 1 , "Freeman's cucumber» absorbant plus et «Persian Small Type moins le potassium que les autres variétés «Doublon » et « Védrantais».

\section{e) Magnésium et calcium}

La solution s'appauvrit surtout en magnésium dans la fin de l'essai 2. On n'observe pas d'effet notable de la tempéra- ture mais par contre, dans l'essai 1, «Freeman's cucumber » absorbe plus de magnésium que les autres variétés. L'évolution du taux de calcium dans la solution nutritive ne permet pas de noter d'effet caractéristique.

\section{Composition de la matière sèche des plantes}

Le tableau 5 rapporte la composition minérale des racines et de la partic aćrienne à la fin du $2^{\mathrm{e}}$ essai.

L'effet de la température est négligeable sur la composition minérale des racines et des parties aériennes des plantes: seules la teneur en manganèse des racines et la teneur en phosphore du feuillage augmentent quand on applique des températures de plus en plus élevées au niveau des racines.

L'influence varićtale est réduite avec une tendance de la variétć «Persian Small Type» a ĉtre moins riche en éléments minéraux (azote, phosphore et potassium) dans les racines. Mais toutes les varićtés, à toutes les températures, ont un système racinaire particulièrement riche en manganèse, puisque le pourcentage de manganèse est environ 3 fois plus élevé dans les racines que dans la partic aérienne.

\section{DISCUSSION}

Nos essais n'ont porté que sur de jeunes plants, avant floraison et fructification; nos observations et conclusions se limitent donc au début de la croissance végétative du melon.

Dans notre dispositif expérimental, nous ne disposions que d'un seul bac thermostaté par température ; aussi seules les comparaisons variétales à une température donnée ont pu être analysées statistiquement, lorsque les observations étaient faites plante par plante (cas des caractéristiques de la croissance, tabl. 2)

Les résultats observés montrent clairement une interaction température racinaire-rayonnement global dans le comportement des variétés de melon. Quand nous maintenons une température élevée au niveau des racines, nous retrouvons l'interaction déjà observée dans la gamme des basses températures (RISSER et al., 1978). Par ailleurs, nous pouvons noter que la croissance des plantes de melon et leur nutrition minérale se déroulent normalement quand la température racinaire reste constamment supérieure à la température de l'air ambiant.

\section{A. Effet des températures et du rayonnement sur le comportement variétal}

$\mathrm{Si}$, dans l'essai 2, les variétés n'ont pas réagi de façon significativement différente, dans l'essai 1 , par contre, les variétés de type "Charentais » se distinguent des 2 variétés "Freeman's cucumber » et "Persian Small Type » qui se sont comportées différemment l'une de l'autre.

"Doublon" et "Védrantais» sont 2 variétés de type "Charentais", seul type variétal cultivé sous abri en France. Ces 2 variétés ont réagi de façon similaire dans les 2 essais, aussi nous les avons regroupées pour présenter le rapport des valeurs moyennes des 2 essais et des températures extrêmes pour les caractères les plus importants étudiés (tabl. 6).

Dans l'ensemble, l'effet rayonnement est plus important que l'effet température : le caractère sur lequel il agit le plus est le poids sec des plantes, l'effet étant particulièrement net pour les racines. 
TABLEAU 5

Composition minérale de la matière sèche des plantes ( $g$. p. cent).

Mineral content of plant dry matter (percentage dry weight basis)

\begin{tabular}{|c|c|c|c|c|c|c|}
\hline $\begin{array}{l}\text { Effet température } \\
\text { (toutes variétés confondues) }\end{array}$ & $\mathbf{N}$ & $\mathbf{P}$ & K & $\mathrm{Ca}$ & $\mathrm{Mg}$ & $\mathrm{Mn}$ \\
\hline \multicolumn{7}{|l|}{ Feuillage } \\
\hline $22^{\circ}$ & 5,7 & 0,83 & 7,6 & 4,75 & 0,42 & 0,019 \\
\hline $26^{\circ}$ & 4,9 & 0,87 & 7,9 & 5,00 & 0,47 & 0,019 \\
\hline $30^{\circ}$ & 5,3 & 0,90 & 7,6 & 5,00 & 0,43 & 0,017 \\
\hline $34^{\circ}$ & 4,3 & 0,96 & 7,9 & 5,08 & 0,47 & 0,018 \\
\hline Racine & 3.6 & 0.79 & 9.5 & 0.41 & 0.23 & 0.032 \\
\hline $26^{\circ}$ & $\begin{array}{l}3,0 \\
3.8\end{array}$ & $\begin{array}{l}0.87 \\
0.80\end{array}$ & $\begin{array}{r}7,5 \\
10,1\end{array}$ & $\begin{array}{l}0,41 \\
0,43\end{array}$ & $\begin{array}{l}0,23 \\
0.29\end{array}$ & $\begin{array}{l}0,032 \\
0,050\end{array}$ \\
\hline $30^{\circ}$ & 3.5 & 0.76 & 9,5 & 0,44 & 0.18 & 0,064 \\
\hline $34^{\circ}$ & 3,6 & 0,74 & 9,5 & 0,44 & 0,19 & 0,073 \\
\hline
\end{tabular}

Effet variétal

(toutes températures confondues)

\begin{tabular}{llllllll}
\hline Feuillage & & & & & & \\
& Doublon & 5,4 & 0,97 & 7,9 & 5,3 & 0,47 & 0,019 \\
& Freeman's & 4,8 & 0,86 & 7,5 & 4,9 & 0,46 & 0,020 \\
& Persian & 4,9 & 0,84 & 7,6 & 4,7 & 0,43 & 0,016 \\
Racine & Védrantais & 5,1 & 0,90 & 8,0 & 5,0 & 0,43 & 0,019 \\
& & & & & & 0,0 \\
& Doublon & 4,7 & 0,83 & 10,4 & 0,48 & 0,20 & 0,060 \\
& Freeman's & 3,4 & 0,77 & 9,2 & $\mathbf{0 , 5 0}$ & 0,19 & 0,050 \\
& Persian & 2,9 & 0,68 & 8,7 & 0,50 & 0,21 & 0,055 \\
& Védrantais & 3,5 & 0,81 & 10,3 & $\mathbf{0 , 5 1}$ & 0,28 & 0,055 \\
\hline
\end{tabular}

TABLEAU 6

Effet comparé du rayonnement et de la température racinaire sur le type "Charentais " ( Doublon » et "Védrantais », confondus). Influence of radiation and root temperature on the growth of "Charentais" type ("Doublon" and "Védrantais" confounded).

\begin{tabular}{|c|c|c|c|}
\hline \multirow[b]{2}{*}{ Rapport } & \multirow{2}{*}{$\begin{array}{l}\text { Effet du rayonnement } \\
\frac{\text { Essai } 2}{\text { Essai } 1}\end{array}$} & \multicolumn{2}{|c|}{ Effet de la température } \\
\hline & & $\frac{30^{\circ} \mathrm{C}}{18^{\circ} \mathrm{C}}$ Essai 1 & $\frac{34^{\circ} \mathrm{C}}{22^{\circ} \mathrm{C}}$ Essai 2 \\
\hline Poids sec du feuillage & 2,0 & 1,7 & $1, \mathbf{1}$ \\
\hline Poids sec des racines & 3,2 & 1,3 & 0,8 \\
\hline Nombre de feuilles sur tige principale ( $14 \mathrm{j}$ après début & & & \\
\hline essai) & 1,3 & 1,2 & 1,0 \\
\hline Nombre de ramifications en fin d'essai & 1,8 & 2,1 & 1,2 \\
\hline Surface foliaire & & 1,8 & \\
\hline Rapport poids sec racine/feuillage & 1,5 & 0,8 & 0,7 \\
\hline Teneur en matière sèche du feuillage & 1,0 & 0,9 & 0,9 \\
\hline Consommation hydrique totale & 1,7 & 1,3 & 1,2 \\
\hline $\begin{array}{l}\text { Teneur en matière minérale de la matière sèche de la } \\
\text { racine }\end{array}$ & & 2,3 & \\
\hline
\end{tabular}

Lorsque le rayonnement, et donc la photosynthèse, est faible (essai 1), on observe un effet de la température du substrat important : de $18^{\circ}$ à $30^{\circ} \mathrm{C}$, l'augmentation de la température entraîne une augmentation du poids sec des racines et du feuillage, du nombre de feuilles sur la tige principale, du nombre des ramifications et, plus légèrement, du rapport des poids secs du système aérien et des racines; seule la teneur en matière sèche du système aérien diminue (tabl. 2). Rappelons que les durées respectives des essais 2 et 1 ont été de 14 et $18 \mathrm{j}$ et que l'effet favorable du rayonnement sur les poids secs et sur le nombre de ramifications aurait été encore mieux mis en évidence si les essais avaient eu la même durée.

Par contre, quand le rayonnement est important (essai 2), on constate que l'augmentation de la température du substrat entre $22^{\circ}$ et $34^{\circ} \mathrm{C}$ a peu d'effet : il paraît y avoir surtout un effet dépressif sur le poids sec des racines et leur importance relative par rapport au feuillage.

En ce qui concerne les 2 autres variétés, dans l'essai 1, «Freeman's cucumber» a réagi favorablement aux fortes températures du substrat ; chez "Persian Small Type ", au contraire, la température optimum du substrat se situe à $22^{\circ} \mathrm{C}$; au-delà, on constate un effet dépressif de l'augmentation de température sur le poids sec, la surface foliaire et la teneur en matière sèche du système aérien.

Il est intéressant de noter qu'en période de faible éclairement, «Persian Small Type » présente des décolorations et «Freeman's cucumber» montre un aspect normal 
aux faibles températures (RISSER et al., 1979). Les réactions aux faibles et fortes températures racinaires semblent donc liées.

Aussi bien « Freeman's cucumber» que «Persian Small Type " ont un rapport feuillage/racine supérieur et une consommation hydrique par $\mathrm{mm}^{2}$ de surface inférieure à ceux des 2 autres variétés ; ces facteurs ne peuvent donc pas expliquer leur comportement mutuel si différent.

A toutes les températures, «Persian Small Type » a le système racinaire le moins abondant et est la variété la moins riche en matière sèche alors que "Freeman's cucumber » est la variété la plus riche.

En période de faible rayonnement, «Freeman's cucumber » absorbe le mieux phosphate, nitrate, potassium et magnésium alors qu'au contraire «Persian Small Type » se caractérise par une faible absorption des nitrates et du potassium. En période de fort rayonnement par contre, «Persian Small Type » semble absorber mieux l'ensemble des éléments minéraux que les autres variétés alors que "Freeman's cucumber" ne se distingue plus de «Doublon » et «Védrantais ». Il est curieux toutefois de constater (tabl, 5) que ce sont les 2 variétés « Doublon » et « Védrantais » qui ont la matière sèche la plus riche en éléments minéraux.

Ces études sur la nutrition minérale en liaison avec la photosynthèse méritent d'être reprises dans des conditions expérimentales plus précises, sur des périodes de temps ne faisant pas intervenir les phénomènes de croissance et dans des conditions de rayonnement contrôlées; ainsi il serait intéressant d'étudier si les différences observées au niveau de la nutrition azotée dépendent essentiellement de l'absorption de l'azote par les racines ou de la nitrate réductase, enzyme qui joue un rôle essenticl dans la réduction des nitrates en acides aminés.

En résumé, il ressort que, pour de jeunes plantes de melon, le chauffage du sol (au-delà de $18^{\circ} \mathrm{C}$ ) ne favorise la croissance qu'en période de faible rayonnement; notons toutefois que, dans nos essais, la température de l'air était maintenue inférieure à environ $30^{\circ} \mathrm{C}$.

\section{B. Evolution de la solution. Nutrition minérale}

L'évolution du $\mathrm{pH}$ dépend assez peu de la température des racines et du rayonnement global mais résulte surtout du stade physiologique de la plante. En effet, au début des 2 essais, il apparaît une acidification liée à une moindre absorption de nitrates. Par contre, quand la croissance de la plante s'accélère, la solution nutritive a tendance à s'alcaliniser en liaison avec une plus forte absorption de nitrates. Cette évolution a été aussi observée par MigUel (1981) qui étudiait l'influence de l'azote nitrique et de l'azote ammoniacal sur la croissance du melon; elle peut s'expliquer par le métabolisme des nitrates dans la racine. La réduction des nitrates en ammonium conduit à une forte production d'ions hydroxyles dont une fraction diffuse dans la solution nutritive, provoquant l'augmentation di $\mathrm{pH}$.

L'interprétation de l'évolution des solutions entre chaque renouvellement est difficile car toutes les plantes n'ont pas le même poids, ni la même consommation hydrique et le rayonnement peut varier au cours de chaque période.

L'observation du tableau 4 montre bien que, dans nos essais, le rayonnement global a été plus important que la température racinaire pour l'absorption différentielle des éléments minéraux. Les forts rayonnements favorisent en général l'absorption, ceci étant particulièrement net pour l'absorption de $\mathrm{PO}_{4} \mathrm{H}_{2}^{-}$.
La teneur en phosphore de la partie aérienne est une fonction croissante de la température des racines. Des observations comparables ont été faites par de nombreux auteurs (CORNILlON, 1980). Elles s'expliquent par une action de la température sur le transfert du phosphore des racines vers le feuillage.

En période de fort rayonnement, la photosynthèse intense provoque des besoins importants en éléments minéraux ; par contre, aux périodes de l'année où le rayonnement global est faible et irrégulier, la photosynthèse est variable d'où une absorption irrégulière d'éléments tels que le potassium et le calcium. En effet, l'absorption des éléments minéraux est un phénomène actif qui dépend de la fourniture d'énergie par la respiration des racines en liaison avec la quantité de substrat disponible.

\section{Effet d'une température des racines supérieure à celle de l'air}

Le $2^{\mathrm{e}}$ essai nous a permis de constater que la croissance du melon restc normale quand la température de tout le système racinaire est maintenue à une valeur supérieure à celle de l'air ambiant, dans les limites de la durée de l'essai, soit $14 \mathrm{j}$. En effet, dans cet essai, les maxima de température de l'air étaient inférieurs de $1^{\circ}$ à $10^{\circ} \mathrm{C}$ à la plus forte température des racines. Toutefois, les organes aériens de la plante, en particulier les feuilles, peuvent avoir des températures largement supérieures à celles de l'air en période de fort rayonnement. D'après LEVITT (1980), la température d'une feuille peut être supérieure à $15^{\circ} \mathrm{C}$ environ à celle de l'air ambiant.

\section{CONCLUSION}

Chez de jeunes plantes de melons, les essais réalisés confirment les observations faites dans une gamme de températures racinaires plus basses, à savoir qu'au-dessus de $18{ }^{\circ} \mathrm{C}$ le rayonnement global est un facteur limitant de la croissance du melon plus important que la température racinaire et qu'il existe une forte interaction entre ces 2 facteurs et la variété cultivée (RISSER et al., 1978). Le maintien des racines à des températures supérieures à celles de l'air ne semble pas entraîner d'anomalie de croissance.

Dans nos essais en période de fort rayonnement, la température racinaire n'a eu aucun effet appréciable; par contre, au printemps, en période de faible rayonnement, l'élévation de la température racinaire a favorisé la croissance des plantes, la variété «Persian Small Type » exceptée.

De fortes différences variétales existent en effet en période de faible rayonnement : ainsi, «Freeman's cucumber" profite mieux de l'augmentation de la température racinaire que les types «Charentais», «Doublon» et "Védrantais », alors qu'au-dessus de $22^{\circ} \mathrm{C}$, l'augmentation de la température racinaire est nuisible à la croissance de «Persian Small Type ». Tout essai de chauffage du sol doit donc tenir compte du rayonnement global et de la variété cultivée.

La nutrition minérale mériterait d'être étudiée d'une façon plus approfondie pour savoir dans quelle mesure elle est sensible aux modifications de la température racinaire et du rayonnement et pour déterminer par là son rôle dans l'influence de ces paramètres sur la croissance du melon. 


\section{RÉFÉRENCES BIBLIOGRAPHIQUES}

C.I.I., 1973. Méthodes de référence pour la détermination des éléments minéraux dans les végétaux. Oléagineux, 8 (2), 87-92. Cornillon P., 1980. Etude bibliographique. Incidence de la température des racines sur la croissance et le développement des plantes. Ann. agron., 31 (1), 63-84.

Cornillon P., Boudon M., 1979. Influence de la température des racines sur la croissance des laitues et des tomates. Incidence sur la composition lipidique. Ann. agron., 30 (5), 431-442.

Levitt J., 1980. IV. High temperature stress, 347-393. In « Responses of plants to environmental stresses », A.P. New York, $2^{\mathrm{e}}$ éd., $471 \mathrm{p}$.
Miguel F., 1981. Influence de la température racinaire sur l'absorption de l'azote nitrique ou ammoniacal par le melon. Diplôme d'Etudes Approfondies, Université des Sciences et Techniques du Languedoc, Académie de Montpellier, $57 \mathrm{p}$.

Risser G., 1979. Varietal difference in growth of melon (Cucumis melo L.) at low temperature. Rep. Cucurbit. Genet. Coop., 2, 23. Risser G., Cornillon P., Rode J. C., Auge M., 1978. Effet de la température des racines sur la croissance de jeunes plants de diverses variétés de melon (Cucumis melo L.). Ann. agron., 29 (5), $453-473$. 